\title{
Increased hippocampal-prefrontal functional connectivity in insomnia
}

Jeanne Leerssen ${ }^{\mathrm{a}}$, Rick Wassing ${ }^{\mathrm{a}}$, Jennifer R. Ramautar ${ }^{\mathrm{a}}$, Diederick Stoffers ${ }^{\mathrm{a}}$, Oti Lakbila-Kamal ${ }^{\mathrm{a}}$, Joy Perrier $^{\mathrm{a}, \mathrm{b}}$, Jessica Bruijel ${ }^{\mathrm{a}}$, Jessica C. Foster-Dingley ${ }^{\mathrm{a}}$, Moji Aghajani ${ }^{\mathrm{c}}$, Eus J.W. van Someren ${ }^{\mathrm{a}, \mathrm{c}, \mathrm{d}}$

${ }^{a}$ Department of Sleep and Cognition, Netherlands Institute for Neuroscience, Meibergdreef 47, 1105 BA, Amsterdam, The Netherlands

${ }^{\mathrm{b}}$ Normandie Univ, UNICAEN, INSERM, COMETE, 2 rue des Rochambelles, 14000 Caen cedex 5, France

${ }^{\mathrm{c}}$ Department of Psychiatry, VU medical center, Oldenaller 1, 1081 HJ, Amsterdam, The Netherlands;

${ }^{\mathrm{d}}$ Department of Integrative Neurophysiology, Neuroscience Campus, VU University and Medical Center, de Boelelaan $1081 \mathrm{HV}$, Amsterdam, The Netherlands.

Corresponding author: Jeanne Leerssen, Netherlands Institute for Neuroscience, Meibergdreef 47, 1105 BA, Amsterdam, The Netherlands, +310205661142, j.leerssen@nin.knaw.nl 


\begin{abstract}
Insomnia Disorder (ID) is the second-most common mental disorder and has a far-reaching impact on daytime functioning. A meta-analysis indicates that, of all cognitive domains, declarative memory involving the hippocampus is most affected in insomnia. Hippocampal functioning has consistently been shown to be sensitive to experimental sleep deprivation. Insomnia however differs from sleep deprivation in many aspects, and findings on hippocampal structure and function have been equivocal. The present study used both structural and resting-state functional Magnetic Resonance Imaging in a larger sample than previously reported to evaluate hippocampal volume and functional connectivity in ID. Included were 65 ID patients (mean age $=48.3 \mathrm{y} \pm 14.0,17$ males) and 65 good sleepers (mean age $=44.1 \mathrm{y} \pm 15.2,23$ males). Insomnia severity was assessed with the Insomnia Severity Index (ISI), subjective sleep with the Consensus Sleep Diary (CSD) and objective sleep by two nights of polysomnography (PSG). Seed-based analysis showed a significantly stronger connectivity of the bilateral hippocampus with the left middle frontal gyrus in ID than in controls $(p=$ .035 , cluster based correction for multiple comparisons). Further analyses across all participants moreover showed that individual differences in the strength of this connectivity were associated with insomnia severity (ISI, $r=.371, p=9.3 \mathrm{e}-5$ ) and with subjective sleep quality (CSD sleep efficiency, $r$ $=-.307, p=.009)$ (all $p$ FDR-corrected). Hippocampal volume did not differ between ID and controls. The findings indicate more severe insomnia and worse sleep quality in people with a stronger functional connectivity between the bilateral hippocampus and the left middle frontal gyrus, part of a circuit that characteristically activates with maladaptive rumination and deactivates with sleep.
\end{abstract}

Keywords: insomnia, sleep, hippocampus, middle frontal gyrus, resting-state fMRI, functional connectivity 


\section{Introduction}

Insomnia disorder (ID) is the second-most common mental disorder (Wittchen et al., 2011). ID is characterized by persistent difficulties with initiating sleep, maintaining sleep or early-morning awakening, that are subjectively associated with daytime dysfunction and distress (DSM-5, American Psychiatric Association, 2013). About 10\% of the population meet the clinical criteria of ID (Morin et al., 2015). ID has an extensive impact on mental and physical health. ID moreover increases the risk of other mental adversities including depression, anxiety disorders, bipolar disorder and suicide (Pigeon, Bishop, and Krueger, 2017), as well as somatic conditions including obesity (Hargens, Kaleth, Edwards, and Butner, 2013), diabetes (Hargens et al., 2013), and cardiovascular disease (Palagini et al., 2013). Theories and treatments have mostly assumed that ID involves reversible psychological mechanisms. The recent identification of risk genes for ID (Hammerschlag et al., 2017) however make brain structural and functional involvement in the disorder likely, as is supported by somewhat equivocal findings in neuroimaging studies (Spiegelhalder et al., 2015).

Which brain structures should be investigated with respect to chronically disturbed sleep? Previous experimental studies established a particular sensitivity of the hippocampus to sleep loss (Kreutzmann, Havekes, Abel, and Meerlo, 2015), a subcortical structure that plays an important role in learning and memory, encoding of spatial information and emotion regulation (Phelps, 2004; Kreutzmann et al., 2015). Animal studies indicate that a prolonged period of sleep deprivation interferes with hippocampal neurogenesis and cell proliferation (Meerlo et al., 2009). Reduced neurogenesis and proliferation could affect both local structural and functional integrity as well as the interaction of the hippocampus with other brain areas. Experimental sleep deprivation (Yoo et al., 2007) and sleep fragmentation (Van Der Werf et al., 2009) in humans has likewise been shown to interfere with subsequent hippocampal functioning. These findings, combined with the fact that, of all cognitive domains, declarative memory involving the hippocampus is most affected in insomnia (Fortier-Brochu, Beaulieu-Bonneau, Ivers, and Morin, 2012), underline the importance of evaluating hippocampal structure and function in insomnia.

Findings on abnormal hippocampal structure in insomnia are mixed. Some studies reported reduced hippocampal volume in insomnia patients (Riemann et al., 2007; Joo, Kim, Suh, and Hong, 
2014) or a negative associations between hippocampal volume and subjective sleep quality (Koo et al., 2017). In contrast, other studies did not find any differences in hippocampal volume between ID patients and controls (Winkelman et al., 2010; Noh et al., 2012). These contrasting results could for example be due to differences in methodology to outline the hippocampus and differences in insomnia duration (Noh et al., 2012). More importantly, the studies described above typically had a sample size of 20-30 participants per group, thus only had the statistical power to detect large effect sizes (Spiegelhalder et al., 2015). Therefore, the studies may have missed small to moderate effects that can still be of functional relevance.

Abnormal hippocampal functional connectivity in insomnia has been reported in two studies. Whole brain connectivity analysis showed increased functional connectivity between the left hippocampus and the left orbital part of the superior frontal gyrus in insomnia patients (Li et al., 2017). However, the participants were instructed to keep their eyes closed during the scan, which increases the risk of falling asleep (Tagliazucchi and Laufs, 2014), which in turn can influence the results. Moreover, exploratory results have to be interpreted with some caution, in case no correction for multiple comparison is applied. Another study on resting-state functional connectivity in insomnia used seed-based analysis of the Default Mode Network (DMN) (Regen et al., 2016). Hippocampal connectivity was not significantly different between ID patients and controls, nevertheless, the findings suggested relevance of individual differences in sleep complaints. Individuals with stronger connectivity between the hippocampus and DMN nodes had a lower sleep efficiency, less Rapid Eye Movement (REM) sleep and a longer sleep-onset latency. Again, no correction for multiple comparisons was applied.

In conclusion, studies on insomnia so far equivocally suggest a possible smaller hippocampal volume and, in contrast, a possible stronger functional connectivity between hippocampal and prefrontal areas. Limitations of the studies are their relatively small sample sizes and the use of exploratory approaches without correcting for multiple comparisons. The current study aimed for more definite conclusions about hippocampal involvement in insomnia, by means of a large sample ( $N=130,65$ ID patients and 65 controls), by evaluating both hippocampal volume and functional connectivity, and by evaluating their associations with both objective and subjective sleep. 


\section{Methods}

\subsection{Participants}

Participants were recruited through advertisements and via the Netherlands Sleep Registry (www.sleepregistry.nl) (Benjamins et al., 2017). Inclusion criteria included an age between 18 and 70 years and, for the participants with ID, fulfillment of the diagnostic criteria according to the Diagnostic and Statistical Manual of Mental Disorders, Fifth Edition (American Psychiatric Association, 2013). Exclusion criteria were: a current diagnosis of sleep apnea, restless leg syndrome, narcolepsy or any other neurological, psychiatric or somatic disorders; the use of sleep medication in the 2 months prior to the study; shiftwork; or any MRI contraindication such as non-MR compatible metal implants, claustrophobia or pregnancy. Of the participants admitted to the study, two were excluded from further analyses due to excessive head motion during the MRI scan $(>3 \mathrm{~mm}$ head displacement); two more were excluded from further analyses because video-monitored eye closures made them suspect of falling asleep during the scan (see 2.2); and a final two more because polysomnography suggested subclinical yet visible sleep related breathing disturbances in one and periodic leg movements during sleep in the other. The final sample included 65 ID participants (age $M \pm S D=48.3 \mathrm{y} \pm 14.0$, range $=21-69 \mathrm{y}, 17$ males $)$ and 65 control participants without sleep problems (age $M \pm S D=44.1 \mathrm{y} \pm 15.2$, range $=22-70 \mathrm{y}, 23$ males). Assessments were part of more elaborate studies that were approved by the ethical board of the VU medical center or the University of Amsterdam. All participants gave informed consent.

\subsection{Procedure}

Participants were scanned at the Spinoza Centre for Neuroimaging in Amsterdam at two different sites on MRI scanners of the same brand and type (Philips Achieva 3T; Amsterdam Medical Center, $N_{I D}=36, N_{\text {control }}=36$; University of Amsterdam, $\left.N_{I D}=29, N_{\text {control }}=29\right)$. On assessment days, participants were asked to refrain from alcohol and drugs. Participants were not allowed to consume caffeinated beverages at least six hours before the MRI scan. Scans were made between 9:00 and 20:00 $\mathrm{hr}$ (there were no differences in time of the day the scan was made between ID and controls 
$t(128)=.06, p=.952)$. For resting-state (RS) fMRI, participants were instructed to look at a fixation cross for 12 minutes, lie still, keep their eyes open, think of nothing in particular, and try not to fall asleep. A representative sample of $56 \%$ of the participants ( 74 out of $132, N_{I D}=37, N_{\text {control }}=37$ ), were video-monitored during scanning, allowing us to evaluate the success of the instructions to the participants. Of these 74 participants, four participants $\left(N_{I D}=3, N_{\text {control }}=1\right)$ closed their eyes for longer than a natural blink. Of these four participants, two insomniacs did so once, and immediately opened them on being probed by the researchers by pushing the intercom button that produced a click in the participant's headphone. The two other participants, one from the insomnia group and one from the control group, closed their eyes multiple times for longer than 5 seconds and were therefore excluded from further analyses (see section 2.1). In the evening, participants were fitted with highdensity electroencephalography nets (HD-EEG, 256-channel Electrical Geodesic Inc., Eugene, OR, Net Amps 300 amplifier, input impedance: $200 \mathrm{M} \Omega$, A/D converter: 24 bits) and stayed overnight in the sleep-lab for two consecutive nights, adhering to their habitual bedtime and wake up time.

\subsection{Sleep parameters}

Insomnia severity was assessed using the Insomnia Severity Index (Bastien, Vallières, and Morin, 2001). Higher scores indicate more severe insomnia. Subjective sleep was measured using the Consensus Sleep Diary (CSD) (Carney et al., 2012), filled out for 9 consecutive days. Average values were calculated for the four most common sleep variables: sleep onset latency (SOL), wake after sleep onset (WASO, total duration of wakefulness after sleep onset), total sleep time (TST, time in bed spent actually asleep) and sleep efficiency (SE, proportion of total sleep time relative to the time in bed). The objective counterparts of the four parameters were determined from the second night of two consecutive nights of sleep laboratory PSG, using high-density electroencephalography.

\subsection{Image acquisition}

MRI data were acquired using a 3-Tesla MRI scanner with a 32-channel head coil (Philips Achieva, Best, Netherlands). Resting state fMRI images were acquired using gradient-echo planar 
imaging (EPI) with repetition time (TR)/ echo time (TE) $=2500 / 28 \mathrm{~ms}$, voxel size $=2.5 \mathrm{~mm}^{3}$, interslice gap $=10 \%, 43$ slices, field of view $(\mathrm{FOV})=240 \times 240 \mathrm{~mm}$, flip angle $=77.2^{\circ}$. In 12 minutes, 288 volumes were acquired. An anatomical T1 weighted image was made as a reference for the RS images $\left(\mathrm{TR} / \mathrm{TE}=8.3 / 3.8 \mathrm{~ms}\right.$, voxel size $=1 \mathrm{~mm}^{3}, 220$ slices, $\mathrm{FOV}=240 \times 188 \mathrm{~mm}$, flip angle $\left.=8^{\circ}\right)$. The homogeneity of the magnetic field was assessed by acquiring a b-zero field map (TR/TE=11/3 $\mathrm{ms}$, voxel size $=2 \mathrm{~mm}^{3}, 128$ slices, FOV $=256 \times 208 \mathrm{~mm}$, flip angle $=8^{\circ}$ ). The same parameters were used at both scanning sites.

\section{$2.5 \quad$ Image preprocessing}

Images were processed using FEAT (FMRI Expert Analysis Tool) Version 6.00, part of FSL (version 5.0.9, FMRIB https://fsl.fmrib.ox.ac.uk/fsl/fslwiki). The following preprocessing steps were performed for the functional images: 1). Brain extraction using BET (Smith, 2002), 2). The first 5 volumes were discarded to correct for signal instabilities, 3). Motion correction using MCFLIRT (Jenkinson, Bannister, Brady, and Smith, 2002), 4). B-zero fieldmap correction (https://osf.io/hks7x), 5). Spatial smoothing (FWHM $=5 \mathrm{~mm}), 6$ ). The ICA-AROMA algorithm was used to automatically remove motion artifacts (Pruim et al., 2015), 7). Regression of nuisance variables such as mean white matter and CSF signal, 8). High-pass filtering (cut-off $100 \mathrm{~s}$ ) and 9). Transformation matrices for linear registration were obtained by Boundary Based Registration of the EPI scan tot the participants anatomical scan combined with a linear transformation of the anatomical scan to the $2 \mathrm{~mm}^{3} \mathrm{MNI}$ template (FLIRT, Jenkinson and Smith, 2001; Jenkinson et al., 2002).

\subsection{Functional connectivity}

The primary, seed-based, analysis addressed group differences in hippocampal connectivity. For each participant, a region of interest (ROI) binary mask of the left and right hippocampus was obtained using FIRST (Patenaude, Smith, Kennedy, and Jenkinson, 2011). The mean Blood Oxygen Level Dependent (BOLD) time series was extracted from these ROIs, by averaging the BOLD signal across all voxels inside the ROI. Subject-level general linear model analyses (GLM) were carried out 
using FEAT (FMRI Expert Analysis Tool, version 6.00, part of FSL) with mean hippocampal BOLD time course (left and right) as predictor variables. Three contrasts were obtained to determine voxels that were positively correlated with the seed regions: left hippocampus, right hippocampus and bilateral hippocampus. As a result, whole-brain maps were created for the left-, right and bilateral hippocampal connectivity with all other voxels in the brain. Group differences in these connectivity maps were tested with a linear mixed effect regression model at each voxel (FLAME stage 1 and 2, Beckmann, Jenkinson, and Smith, 2003; Woolrich et al., 2004), using generalized least squares with a local estimate of random effects variance. Automatic outlier de-weighting was used to eliminate the possible influence of outlier observations (Woolrich, 2008). Demeaned values of age, time of day, and dummy coded sex and scanner site were included in the model as covariates. Cluster-based correction was used to correct for multiple comparisons $(Z>3.1, p<.05)$ using Gaussian Random Field Theory (Worsley, 2001).

In case of significant group differences in hippocampal functional connectivity, secondary analyses were performed to explore involvement of different subjective and objective sleep measures, and to assess whether group differences involved gradual or discrete differences across all participants (ID patients and controls). For each individual, the strength of connectivity between the hippocampal seed and significant clusters was obtained with FSL Featquery, extracting the mean Z-values representing an individual's hippocampal connectivity strengths with significant clusters. These connectivity strengths were correlated with the subjective and objective sleep parameters described above, with the use of partial correlation analyses in R (R Core Team, 2016). Models were adjusted for age, sex, time of day and scanner site. The reported $p$-values are corrected for multiple comparison with the use of False Discovery Rate (FDR) correction (Benjamini and Hochberg, 1995).

\subsection{Hippocampal volume}

Group differences in left, right and bilateral hippocampal volume were calculated to determine whether structural abnormalities co-occur with the functional abnormalities in the hippocampus in insomnia. The volumes of the left, right and bilateral hippocampus were calculated with FIRST (Patenaude et al., 2011). Total brain volume was estimated with SIENAX (Smith, De Stefano, 
Jenkinson, and Matthews, 2001; Smith et al., 2002). Group differences in left, right and bilateral hippocampal volume between insomnia patients and controls were assessed with linear regression model analysis in R (R Core Team, 2016). Age, sex, total brain volume and scanner site were included in the model as covariates. In case of significant group differences in hippocampal volume, secondary analyses were performed to explore involvement of different subjective and objective sleep measures, and to assess whether group differences involved gradual or discrete differences, using partial correlation analyses as described above for functional connectivity (see 2.6).

\section{Results}

\subsection{Participants}

Table 1 presents the descriptive statistics of the insomnia and control group. ID patients did not differ significantly from controls with respect to age $(t(128)=1.62, \mathrm{p}=.108)$ or $\operatorname{sex}\left(\chi^{2}=0.90, p=\right.$ .342). Compared to the control group, the insomnia group experienced worse sleep on all variables except for objective sleep onset latency (PSG: $t(104)=0.04, p=.972$ ). The ID group had a higher ISI $(t(126)=14.35, p=2.2 \mathrm{e}-16)$, longer subjective SOL $(\mathrm{CSD}: t(96)=4.39, p=3.0 \mathrm{e}-05)$, longer WASO $(\mathrm{CSD}: t(96)=6.69, p=1.5 \mathrm{e}-9$; PSG: $t(104)=3.09, p=.003)$, shorter TST $(\mathrm{CSD}: t(96)=-8.24, p=$ 8.6e-13; PSG: $t(104)=-2.7, p=.007)$, and lower SE (CSD: $t(96)=-8.72, p=8.1 \mathrm{e}-14$; PSG: $t(104)=$ $-3.35, p=.001)$ compared to the control group.

\subsection{Functional connectivity}

As compared to the control group, people with ID showed stronger functional connectivity between the bilateral hippocampus and a cluster of voxels in the left middle frontal gyrus (MFG, Brodmann Area 8, 129 voxels, $\mathrm{p}=.035, \mathrm{Z}_{\max }=4.22, \mathrm{MNI}_{\mathrm{x}, \mathrm{y}, \mathrm{Z}}$ coordinates peak value at $[-26,10,52]$, cluster based correction for multiple comparisons at $\alpha=.05, Z>3.1$ ) (see Figure 1). No group differences reached significance on the contrasts of left and right hippocampal connectivity.

Across all participants, partial correlation analyses with FDR correction for multiple comparisons ( $N=9$ tests) showed that individual differences in the hippocampus-MFG connectivity strength correlated with subjective ratings of insomnia severity $\left(r=.371, p_{\text {corrected }}=9.3 \mathrm{e}-5\right)$, and subjective $\mathrm{SE}$ 
$\left(r=-.307, p_{\text {corrected }}=.009\right)$ (see Figure 2 and Table 2), indicating a worse sleep experience with stronger connectivity. Without correction for multiple comparisons, subjective TST ( $r=-.213$, $\left.p_{\text {uncorrected }}=.036\right)$ and objective WASO $\left(r=.226, p_{\text {uncorrected }}=.021\right)$ also correlated with hippocampusMFG connectivity strength. Stronger connectivity was associated with shorter total sleep time and longer wake time after sleep onset. When the sample was halved to include only the insomnia group, correlations of sleep parameters with hippocampus-MFG connectivity no longer reached significance correcting for multiple comparisons ( $N=9$ tests, all $p>.410$ ), and without correcting for multiple comparison (all $p>.071)$.

\subsection{Hippocampal volume}

Insomnia patients did not differ from controls with respect to bilateral hippocampal volume $\left(M_{I D}\right.$ $\left.=7949 \mathrm{~mm}^{3}, S D=794 ; M_{\text {control }}=7916 \mathrm{~mm}^{3}, S D=831 ; \Delta_{\text {mean }}=33.5 \mathrm{~mm}^{3}, t=0.69, p=.493\right)$, adjusted for age, sex, total brain volume and scanner site. Similarly, separate analyses on left and right hippocampal volume did not show differences between insomnia patients and controls (left: $M_{I D}=$ $3948 \mathrm{~mm}^{3}, S D=388, M_{\text {control }}=3896 \mathrm{~mm}^{3}, S D=468, \Delta_{\text {mean }}=52.4 \mathrm{~mm}^{3}, t=1.12, p=.267 ;$ right: $M_{I D}$ $\left.=4001 \mathrm{~mm}^{3}, S D=484, M_{\text {control }}=4019 \mathrm{~mm}^{3}, S D=539, \Delta_{\text {mean }}=-18.9 \mathrm{~mm}^{3}, t=0.09, p=.931\right)$.

\section{Conclusion and discussion}

Given the hippocampal sensitivity to disturbed sleep, as well as the meta-analytic finding that, of all cognitive domains, declarative memory involving the hippocampus is most affected in insomnia, the current study addressed whether insomnia is related to hippocampal functional connectivity and volume. As compared to controls without sleep complaints, people with insomnia showed an increased connectivity between the hippocampus and part of the left middle frontal gyrus. Follow-up analyses indicated gradual dimensional effects across controls and cases rather than discrete effects related to the absence versus presence of diagnosis: stronger connectivity between these regions was associated with more severe insomnia and with a lower subjective sleep efficiency. Hippocampal volume did not differ significantly between ID and controls. 
Our finding of increased hippocampal connectivity in association with insomnia is in line with two previous reports. Li et al. (2017) reported increased connectivity between the hippocampus and the left orbital part of the superior frontal gyrus in insomnia. Regen et al. (2016) found worse objective sleep efficiency with increasing connectivity between the hippocampus and the ventromedial prefrontal cortex. Our present findings confirm increased connectivity between hippocampus and prefrontal cortex in insomnia, but found this to be most significant for connectivity with the left middle frontal gyrus, which has not previously been implicated in insomnia. Although there are differences in methodology, study population, insomnia severity and sample size between our study and previous studies, the findings concertedly strengthen the possibility that increased hippocampus-prefrontal connectivity is involved in insomnia.

What are the functional correlates of this specific connectivity between hippocampus and middle frontal gyrus? And how could increased hippocampus-MFG connectivity be involved in insomnia, either as a consequence, or as causally contributing to the risk of experiencing sleep complaints? A study of Andrade et al. (2011) gives some insight in the functional correlates of this specific connectivity in light of sleep and wakefulness. The authors conducted a simultaneous EEG-fMRI study in healthy subjects to reveal distinct hippocampal functional connectivity changes across wake and different stages of sleep. Of relevance to our current results was their finding that strong connectivity between the hippocampus and a cluster within the middle/superior frontal gyrus is characteristic of wakefulness while connectivity during sleep was weak. The middle/superior frontal gyrus cluster showing this state dependent change in connectivity overlapped with the cluster we found in our current study. It is tempting to consider the possibility that insomnia involves a relative persistence of wake-related functional connectivity prior to sleep, impeding the transition to sleep. The interpretation that individual differences in the strength of wake-related functional connectivity could causally contribute to the development of disruptions in sleep quality is in line with the finding that the pre-sleep cognitive arousal that characterizes people with insomnia (Sánchez-Ortuño et al., 2011) is already present prior to onset of the disorder (Gregory et al., 2008; Fernández-Mendoza et al., 2010). However, given the strong group difference in subjective, but not objective, sleep onset latency a more interesting interpretation may be that insomnia involves a relative persistence of wake- 
related functional connectivity during sleep, thus contributing to the robust finding that people with insomnia experience part of their sleep as being awake (Krystal, Edinger, Wohlgemuth, and Marsh, 2002). Future simultaneous EEG-fMRI studies may evaluate whether people with insomnia differ with respect to the characteristic sleep onset-related changes in functional connectivity (Tagliazucchi and van Someren, 2017).

Another possible functional correlate of hippocampus-MFG connectivity worth considering in light of insomnia is rumination. Both the hippocampus (Vincent et al., 2006; Buckner, AndrewsHanna, and Schacter, 2008) and the left MFG cluster we here found (Gujar, Yoo, Hu, and Walker, 2010; Chang et al., 2013; Wang et al., 2015a) are deemed part of the default model network (DMN). The DMN is one of the key resting-state networks that deactivates during goal directed behavior, activates during rest and is involved in self-referential mental thought (Mak et al., 2017). Hippocampal integration in the DMN seems particularly prominent during the retrieval of episodic memories (Huijbers, Pennartz, Cabeza, and Daselaar, 2011). Functional connectivity within the DMN at rest has repeatedly been found to correlate with brooding, the maladaptive type of rumination (Freton et al., 2014; Wang et al., 2015b; Lois and Wessa, 2016). It is conceivable that enhanced hippocampus-MFG connectivity could contribute to the brooding type of rumination that is believed to play a role in maintaining insomnia disorder (Carney, Harris, Moss, and Edinger, 2010; Morin et al., 2015). Although not a planned analysis, we had measured trait-like brooding in 82 participants $\left(N_{I D}=40, N_{\text {control }}=42\right)$ (Treynor, Gonzalez, and Nolen-Hoeksema, 2003) on another occasion within a range of $1.2-4.1$ years after the MRI scan. In spite of the distance in time, ID participants still tended to report more brooding than controls $(t(80)=1.76, p=.082)$. Future studies may evaluate brooding and other resting-state cognitions (Diaz et al., 2013; Diaz et al., 2014) immediately following the RSfMRI scan to relate it to hippocampus-MFG connectivity.

A related possibly relevant functional correlate of increased functional connectivity within the DMN concerns its association with resting-state EEG. In a combined EEG-fMRI wake resting-state study, Hlinka et al. (2010) found that individuals with a stronger functional connectivity within the DMN have more power in higher frequencies and less power in lower frequencies of the EEG. 
Interestingly, the very same disbalance has been seen in the wake resting-state EEG of insomnia patients (Colombo et al., 2016).

In the current study, we did not observe differences in hippocampal volume between people suffering from insomnia and matched controls. This finding is in line with two previous studies that also could not detect hippocampal volumes abnormalities in insomnia (Winkelman et al., 2010; Noh et al., 2012). Two other previous studies did however report differences in hippocampal volume between insomnia patients and controls (Riemann et al., 2007; Joo et al., 2014). Methodological differences in defining the hippocampus may be involved in the discrepancy. Riemann et al. (2007) and Joo et al. (2014) manually outlined the hippocampus, whereas in our study we used an automatic tool, FIRST as part of FSL. Although automatic tools tend to overestimate hippocampal volume (Schoemaker et al., 2016), it is still a reliable (Morey et al., 2010; Nugent et al., 2013), and more importantly, a reproducible method (Mulder et al., 2014; Bartel et al., 2017) to estimate hippocampal volume across studies. Our study population also differed from Riemann et al. and Joo et al. in terms of insomnia severity and insomnia duration. The mean ISI in our sample was 16.3 compared to 19.0 in the insomnia group of the study of Riemann et al. The mean insomnia duration in our study was longer (20.2 years) than reported in the studies of Riemann et al. and Joo et al. (11.6 and 8.4 year respectively). However, the sample size in our study was 2 to 8 times larger $(N=130)$ than the other studies ( $N=16$ in Riemann et al., $N=57$ in Joo et al.). It is therefore unlikely that a lack of statistical power could be involved in our inability to replicate previous findings of hippocampal volume abnormalities in insomnia.

Resting state functional connectivity measures tend to change during the process of falling asleep (Tagliazucchi and van Someren, 2017). It is therefore important to discuss the question whether group differences in hippocampus-MFG connectivity might have resulted from group differences in falling asleep during the scan. Current observations make such an explanation unlikely. A representative sample of our participants were video-monitored during scanning and supervisors were instructed to push the intercom button that produced a click in the participant's headphone if their eyes were closed longer than for a natural blink. On suspicion of possible falling asleep we excluded two participants. 
Therefore, it is unlikely that participants fell asleep during the eyes-open assessment, and consequently also that groups differed with respect to falling asleep.

The current study has several limitations. Given our focus on hippocampal connectivity, we have not explored possible group differences in resting-state functional connectivity between other brain areas. Future studies using whole brain approaches or independent components based analysis may give a more comprehensive view of alterations with functional relevance to insomnia, provided that sample sizes give sufficient statistical power to control for false discovery. It should also be mentioned that the interpretation of the current results is somewhat limited because we did not assess performance on declarative memory tasks. It would be interesting to see how memory performance would relate to the connectivity strength between the hippocampus and MFG.

The current study is also limited by the fact that we assumed that our participants represented a homogeneous sample of a single insomnia disorder. It is increasingly considered that insomnia could be a heterogeneous disorder and that inconsistent findings may result if different studies include different proportions of each possibly unrecognized subtypes (Vgontzas, Fernandez-Mendoza, Liao, and Bixler, 2013; Benjamins et al., 2017). Previous research has shown differences in cognitive functioning between insomniacs that have a short $(<6 \mathrm{~h})$ versus normal $(\geq 6 \mathrm{~h})$ sleep duration (Fernandez-Mendoza et al., 2010). It is conceivable that these insomnia subtypes could differ in terms of their hippocampal connectivity patterns. In the current sample, the proportion of insomnia participants with a consistent sleep duration of less than 6 hours on both nights of PSG was $14.3 \%$, which is surprisingly identical to the proportion of consistent short sleep in the large sample of people with insomnia recently reported by Johann et al. (2017). The small proportion does not provide subgroups of sufficient size to address possible specific deviations in people with insomnia that consistently sleep short. Future studies including more cases of this subtype could address this possibility.

Another limitation is that the cross-sectional nature of our study allows us to only speculate about the causal versus consequential interpretation of our results. Although we cannot exclude the possibility that increased hippocampal connectivity specifically with the MFG could result from insomnia, we consider it more likely that pre-existing individual differences in this connectivity 
translate to differences in the risk of experiencing complaints of insomnia. Hippocampal-prefrontal circuits have long been associated with several psychiatric disorders including mood disorders and schizophrenia (Li, Long, and Yang, 2015). Abnormalities in these circuits are thought to increase the vulnerability to develop psychopathology. Future long-term follow-up studies in large cohorts would be required to evaluate whether pre-existing individual differences in hippocampus - MFG connectivity could contribute to the risk of developing insomnia. Apart from these limitations, a major strength of our study is that it has a larger sample size than is usual in neuroimaging studies of insomnia. Another strength is the convergence of findings with subjective and objective measures of insomnia, sleep quality and sleep quantity.

In summary, our findings indicate more severe insomnia and worse sleep quality and quantity in people with a stronger functional connectivity between the bilateral hippocampus and an area within the left middle frontal gyrus, part of a circuit that characteristically activates with maladaptive rumination and deactivates with sleep. 


\section{Acknowledgements}

Conflict of interest: none. This work was supported by the Netherlands Organization of Scientific Research (NWO), The Hague, The Netherlands (ZONMW Neuropsychoanalysis Fund Grant 16.561.0001 and VICI 453.07.001), by the Bial Foundation, S. Mamede do Coronado, Portugal (Grant 253/2012 and 190/2016) and by the European Commission (European Research Council Grant ERC-ADG-2014-671084 INSOMNIA). This publication reflects the views only of the authors, and the Commission cannot be held responsible for any use which may be made of the information contained therein. 


\section{References}

American Psychiatric Association (2013). Diagnostic and statistical manual of mental disorders (DSM-5®): American Psychiatric Pub.

Andrade, K. C., Spoormaker, V. I., Dresler, M., Wehrle, R., Holsboer, F., Samann, P. G., \& Czisch, M. (2011). Sleep spindles and hippocampal functional connectivity in human NREM sleep. Journal of Neuroscience, 31, 10331-10339.

Bartel, F., Vrenken, H., Bijma, F., Barkhof, F., van Herk, M., \& de Munck, J. C. (2017). Regional analysis of volumes and reproducibilities of automatic and manual hippocampal segmentations. PLoS One, 12, e0166785.

Bastien, C. H., Vallières, A., \& Morin, C. M. (2001). Validation of the Insomnia Severity Index as an outcome measure for insomnia research. Sleep Medicine, 2, 297-307.

Beckmann, C. F., Jenkinson, M., \& Smith, S. M. (2003). General multilevel linear modeling for group analysis in FMRI. Neuroimage, 20, 1052-1063.

Benjamini, Y., \& Hochberg, Y. (1995). Controlling the false discovery rate: a practical and powerful approach to multiple testing. Journal of the Royal Statistical Society. Series B (Methodological), 289-300.

Benjamins, J. S., Migliorati, F., Dekker, K., Wassing, R., Moens, S., Blanken, T. F., te Lindert, B. H., Mook, J. S., \& Van Someren, E. J. (2017). Insomnia heterogeneity: Characteristics to consider for data-driven multivariate subtyping. Sleep Medicine Reviews, 36, 71-81.

Buckner, R. L., Andrews-Hanna, J. R., \& Schacter, D. L. (2008). The brain's default network: anatomy, function, and relevance to disease. Annals of the New York Academy of Sciences, 1124, 1-38.

Carney, C. E., Buysse, D. J., Ancoli-Israel, S., Edinger, J. D., Krystal, A. D., Lichstein, K. L., \& Morin, C. M. (2012). The consensus sleep diary: standardizing prospective sleep selfmonitoring. Sleep, 35, 287-302.

Carney, C. E., Harris, A. L., Moss, T. G., \& Edinger, J. D. (2010). Distinguishing rumination from worry in clinical insomnia. Behaviour Research and Therapy, 48, 540-546. 
Chang, C., Liu, Z., Chen, M. C., Liu, X., \& Duyn, J. H. (2013). EEG correlates of time-varying BOLD functional connectivity. Neuroimage, 72, 227-236.

Colombo, M., Ramautar, J. R., Wei, Y., Gomez-Herrero, G., Stoffers, D., Wassing, R., Benjamins, J., Tagliazucchi, E., Van Der Werf, Y. D., Cajochen, C., \& Van Someren, E. J. W. (2016). Wake high-density electroencephalographic spatiospectral signatures of insomnia. Sleep, 39, 10151027.

Diaz, B. A., Van Der Sluis, S., Benjamins, J. S., Stoffers, D., Hardstone, R., Mansvelder, H. D., Van Someren, E. J., \& Linkenkaer-Hansen, K. (2014). The ARSQ 2.0 reveals age and personality effects on mind-wandering experiences. Frontiers in Psychology, 5.

Diaz, B. A., Van Der Sluis, S., Moens, S., Benjamins, J. S., Migliorati, F., Stoffers, D., Den Braber, A., Poil, S.-S., Hardstone, R., \& Van't Ent, D. (2013). The Amsterdam Resting-State Questionnaire reveals multiple phenotypes of resting-state cognition. Frontiers in Human Neuroscience, 7.

Fernandez-Mendoza, J., Calhoun, S., Bixler, E. O., Pejovic, S., Karataraki, M., Liao, D., Vela-Bueno, A., Ramos-Platon, M. J., Sauder, K. A., \& Vgontzas, A. N. (2010). Insomnia with objective short sleep duration is associated with deficits in neuropsychological performance: a general population study. Sleep, 33, 459-465.

Fernández-Mendoza, J., Vela-Bueno, A., Vgontzas, A. N., Ramos-Platón, M. J., OlavarrietaBernardino, S., Bixler, E. O., \& De la Cruz-Troca, J. J. (2010). Cognitive-emotional hyperarousal as a premorbid characteristic of individuals vulnerable to insomnia. Psychosomatic Medicine, 72, 397-403.

Fortier-Brochu, É., Beaulieu-Bonneau, S., Ivers, H., \& Morin, C. M. (2012). Insomnia and daytime cognitive performance: a meta-analysis. Sleep Medicine Reviews, 16, 83-94.

Freton, M., Lemogne, C., Delaveau, P., Guionnet, S., Wright, E., Wiernik, E., Bertasi, E., \& Fossati, P. (2014). The dark side of self-focus: brain activity during self-focus in low and high brooders. Social Cognitive and Affective Neuroscience, 9, 1808-1813.

Gregory, A. M., Willis, T. A., Wiggs, L., Harvey, A. G., \& team, S. (2008). Presleep arousal and sleep disturbances in children. Sleep, 31, 1745-1747. 
Gujar, N., Yoo, S. S., Hu, P., \& Walker, M. P. (2010). The unrested resting brain: sleep deprivation alters activity within the default-mode network. Journal of Cognitive Neuroscience, 22, 1637 1648.

Hammerschlag, A. R., Stringer, S., de Leeuw, C. A., Sniekers, S., Taskesen, E., Watanabe, K., Blanken, T. F., Dekker, K., Te Lindert, B. H. W., Wassing, R., Jonsdottir, I., Thorleifsson, G., Stefansson, H., Gislason, T., Berger, K., Schormair, B., Wellmann, J., Winkelmann, J., Stefansson, K., Oexle, K., Van Someren, E. J. W., \& Posthuma, D. (2017). Genome-wide association analysis of insomnia complaints identifies risk genes and genetic overlap with psychiatric and metabolic traits. Nature Genetics, 49, 1584-1592.

Hargens, T. A., Kaleth, A. S., Edwards, E. S., \& Butner, K. L. (2013). Association between sleep disorders, obesity, and exercise: a review. Nature and Science of Sleep, 5, 27-35.

Hlinka, J., Alexakis, C., Diukova, A., Liddle, P. F., \& Auer, D. P. (2010). Slow EEG pattern predicts reduced intrinsic functional connectivity in the default mode network: an inter-subject analysis. Neuroimage, 53, 239-246.

Huijbers, W., Pennartz, C. M., Cabeza, R., \& Daselaar, S. M. (2011). The hippocampus is coupled with the default network during memory retrieval but not during memory encoding. PLoS One, 6, e17463.

Jenkinson, M., Bannister, P., Brady, M., \& Smith, S. (2002). Improved optimization for the robust and accurate linear registration and motion correction of brain images. Neuroimage, 17, 825841.

Jenkinson, M., \& Smith, S. (2001). A global optimisation method for robust affine registration of brain images. Medical Image Analysis, 5, 143-156.

Johann, A. F., Hertenstein, E., Kyle, S. D., Baglioni, C., Feige, B., Nissen, C., McGinness, A. J., Riemann, D., \& Spiegelhalder, K. (2017). Insomnia with objective short sleep duration is associated with longer duration of insomnia in the Freiburg Insomnia Cohort compared to insomnia with normal sleep duration, but not with hypertension. PLoS One, 12, e0180339. 
Joo, E. Y., Kim, H., Suh, S., \& Hong, S. B. (2014). Hippocampal substructural vulnerability to sleep disturbance and cognitive impairment in patients with chronic primary insomnia: magnetic resonance imaging morphometry. Sleep, 37, 1189-1198.

Koo, D. L., Shin, J. H., Lim, J. S., Seong, J. K., \& Joo, E. Y. (2017). Changes in subcortical shape and cognitive function in patients with chronic insomnia. Sleep Medicine, 35, 23-26.

Kreutzmann, J. C., Havekes, R., Abel, T., \& Meerlo, P. (2015). Sleep deprivation and hippocampal vulnerability: changes in neuronal plasticity, neurogenesis and cognitive function. Neuroscience, 309, 173-190.

Krystal, A. D., Edinger, J. D., Wohlgemuth, W. K., \& Marsh, G. R. (2002). NREM sleep EEG frequency spectral correlates of sleep complaints in primary insomnia subtypes. Sleep, 25, 626-636.

Li, C., Dong, M., Yin, Y., Hua, K., Fu, S., \& Jiang, G. (2017). Abnormal whole-brain functional connectivity in patients with primary insomnia. Neuropsychiatric Disease and Treatment, 13, 427-435.

Li, M., Long, C., \& Yang, L. (2015). Hippocampal-prefrontal circuit and disrupted functional connectivity in psychiatric and neurodegenerative disorders. Biomedical Research International, 2015, 810548.

Lois, G., \& Wessa, M. (2016). Differential association of default mode network connectivity and rumination in healthy individuals and remitted MDD patients. Social Cognitive and Affective Neuroscience, 11, 1792-1801.

Mak, L. E., Minuzzi, L., MacQueen, G., Hall, G., Kennedy, S. H., \& Milev, R. (2017). The default mode network in healthy individuals: A systematic review and meta-analysis. Brain Connectivity, 7, 25-33.

Meerlo, P., Mistlberger, R. E., Jacobs, B. L., Heller, H. C., \& McGinty, D. (2009). New neurons in the adult brain: the role of sleep and consequences of sleep loss. Sleep Medicine Reviews, 13, 187-194. 
Morey, R. A., Selgrade, E. S., Wagner, H. R., 2nd, Huettel, S. A., Wang, L., \& McCarthy, G. (2010). Scan-rescan reliability of subcortical brain volumes derived from automated segmentation. Human Brain Mapping, 31, 1751-1762.

Morin, C. M., Drake, C. L., Harvey, A. G., Krystal, A. D., Manber, R., Riemann, D., \& Spiegelhalder, K. (2015). Insomnia disorder. Nature Reviews Disease Primers, 1, 15026.

Mulder, E. R., de Jong, R. A., Knol, D. L., van Schijndel, R. A., Cover, K. S., Visser, P. J., Barkhof, F., Vrenken, H., \& Alzheimer's Disease Neuroimaging, I. (2014). Hippocampal volume change measurement: quantitative assessment of the reproducibility of expert manual outlining and the automated methods FreeSurfer and FIRST. Neuroimage, 92, 169-181.

Noh, H. J., Joo, E. Y., Kim, S. T., Yoon, S. M., Koo, D. L., Kim, D., Lee, G. H., \& Hong, S. B. (2012). The relationship between hippocampal volume and cognition in patients with chronic primary insomnia. Journal of Clinical Neurology, 8, 130-138.

Nugent, A. C., Luckenbaugh, D. A., Wood, S. E., Bogers, W., Zarate, C. A., Jr., \& Drevets, W. C. (2013). Automated subcortical segmentation using FIRST: test-retest reliability, interscanner reliability, and comparison to manual segmentation. Human Brain Mapping, 34, 2313-2329.

Palagini, L., Maria Bruno, R., Gemignani, A., Baglioni, C., Ghiadoni, L., \& Riemann, D. (2013). Sleep loss and hypertension: a systematic review. Current Pharmaceutical Design, 19, 24092419.

Patenaude, B., Smith, S. M., Kennedy, D. N., \& Jenkinson, M. (2011). A Bayesian model of shape and appearance for subcortical brain segmentation. Neuroimage, 56, 907-922.

Phelps, E. A. (2004). Human emotion and memory: interactions of the amygdala and hippocampal complex. Current Opinion in Neurobiology, 14, 198-202.

Pigeon, W. R., Bishop, T. M., \& Krueger, K. M. (2017). Insomnia as a Precipitating Factor in New Onset Mental Illness: a Systematic Review of Recent Findings. Current Psychiatry Report, $19,44$.

Pruim, R. H., Mennes, M., van Rooij, D., Llera, A., Buitelaar, J. K., \& Beckmann, C. F. (2015). ICAAROMA: A robust ICA-based strategy for removing motion artifacts from fMRI data. Neuroimage, 112, 267-277. 
R Core Team (2016). R: A language and environment for statistical computing. Vienna, Austria: R Foundation for Statistical Computing; 2014.

Regen, W., Kyle, S. D., Nissen, C., Feige, B., Baglioni, C., Hennig, J., Riemann, D., \& Spiegelhalder, K. (2016). Objective sleep disturbances are associated with greater waking resting-state connectivity between the retrosplenial cortex/hippocampus and various nodes of the default mode network. Journal of Psychiatry \& Neuroscience, 41, 295.

Riemann, D., Voderholzer, U., Spiegelhalder, K., Hornyak, M., Buysse, D. J., Nissen, C., Hennig, J., Perlis, M. L., van Elst, L. T., \& Feige, B. (2007). Chronic insomnia and MRI-measured hippocampal volumes: a pilot study. Sleep, 30, 955-958.

Sánchez-Ortuño, M. M., Carney, C. E., Edinger, J. D., Wyatt, J. K., \& Harris, A. (2011). Moving beyond average values: assessing the night-to-night instability of sleep and arousal in DSMIV-TR insomnia subtypes. Sleep, 34, 531-539.

Schoemaker, D., Buss, C., Head, K., Sandman, C. A., Davis, E. P., Chakravarty, M. M., Gauthier, S., \& Pruessner, J. C. (2016). Hippocampus and amygdala volumes from magnetic resonance images in children: assessing accuracy of FreeSurfer and FSL against manual segmentation. Neuroimage, 129, 1-14.

Smith, S. M. (2002). Fast robust automated brain extraction. Human Brain Mapping, 17, 143-155.

Smith, S. M., De Stefano, N., Jenkinson, M., \& Matthews, P. M. (2001). Normalized accurate measurement of longitudinal brain change. Journal of Computer Assisted Tomography, 25, $466-475$.

Smith, S. M., Zhang, Y., Jenkinson, M., Chen, J., Matthews, P., Federico, A., \& De Stefano, N. (2002). Accurate, robust, and automated longitudinal and cross-sectional brain change analysis. Neuroimage, 17, 479-489.

Spiegelhalder, K., Regen, W., Baglioni, C., Nissen, C., Riemann, D., \& Kyle, S. D. (2015). Neuroimaging insights into insomnia. Current Neurology and Neuroscience Report, 15, 9. Tagliazucchi, E., \& Laufs, H. (2014). Decoding wakefulness levels from typical fMRI resting-state data reveals reliable drifts between wakefulness and sleep. Neuron, 82, 695-708. 
Tagliazucchi, E., \& van Someren, E. J. (2017). The large-scale functional connectivity correlates of consciousness and arousal during the healthy and pathological human sleep cycle. Neuroimage, 160, 55-72.

Treynor, W., Gonzalez, R., \& Nolen-Hoeksema, S. (2003). Rumination reconsidered: A psychometric analysis. Cognitive Therapy and Research, 27, 247-259.

Van Der Werf, Y. D., Altena, E., Schoonheim, M. M., Sanz-Arigita, E. J., Vis, J. C., De Rijke, W., \& Van Someren, E. J. (2009). Sleep benefits subsequent hippocampal functioning. Nature Neuroscience, 12, 122-123.

Vgontzas, A. N., Fernandez-Mendoza, J., Liao, D., \& Bixler, E. O. (2013). Insomnia with objective short sleep duration: the most biologically severe phenotype of the disorder. Sleep Medicine Reviews, 17, 241-254.

Vincent, J. L., Snyder, A. Z., Fox, M. D., Shannon, B. J., Andrews, J. R., Raichle, M. E., \& Buckner, R. L. (2006). Coherent spontaneous activity identifies a hippocampal-parietal memory network. Journal of Neurophysiology, 96, 3517-3531.

Wang, H., Zeng, L.-L., Chen, Y., Yin, H., Tan, Q., \& Hu, D. (2015a). Evidence of a dissociation pattern in default mode subnetwork functional connectivity in schizophrenia. Scientific Reports, 5, 14655.

Wang, K., Wei, D., Yang, J., Xie, P., Hao, X., \& Qiu, J. (2015b). Individual differences in rumination in healthy and depressive samples: association with brain structure, functional connectivity and depression. Psychological Medicine, 45, 2999-3008.

Winkelman, J. W., Benson, K. L., Buxton, O. M., Lyoo, I. K., Yoon, S., O'Connor, S., \& Renshaw, P. F. (2010). Lack of hippocampal volume differences in primary insomnia and good sleeper controls: an MRI volumetric study at 3 Tesla. Sleep Medicine, 11, 576-582.

Wittchen, H.-U., Jacobi, F., Rehm, J., Gustavsson, A., Svensson, M., Jönsson, B., Olesen, J., Allgulander, C., Alonso, J., \& Faravelli, C. (2011). The size and burden of mental disorders and other disorders of the brain in Europe 2010. European Neuropsychopharmacology, 21, 655-679.

Woolrich, M. (2008). Robust group analysis using outlier inference. Neuroimage, 41, 286-301. 
Woolrich, M. W., Behrens, T. E., Beckmann, C. F., Jenkinson, M., \& Smith, S. M. (2004). Multilevel linear modelling for FMRI group analysis using Bayesian inference. Neuroimage, 21, 17321747.

Worsley, K. (2001). Statistical analysis of activation images, Functional MRI: an introduction to methods (pp. 251-270): OUP.

Yoo, S. S., Hu, P. T., Gujar, N., Jolesz, F. A., \& Walker, M. P. (2007). A deficit in the ability to form new human memories without sleep. Nature Neuroscience, 10, 385-392. 
Figures

A 10

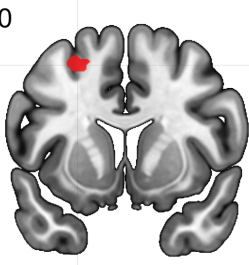

52

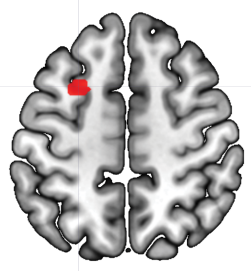

$-26$

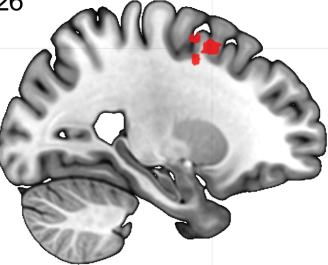

B

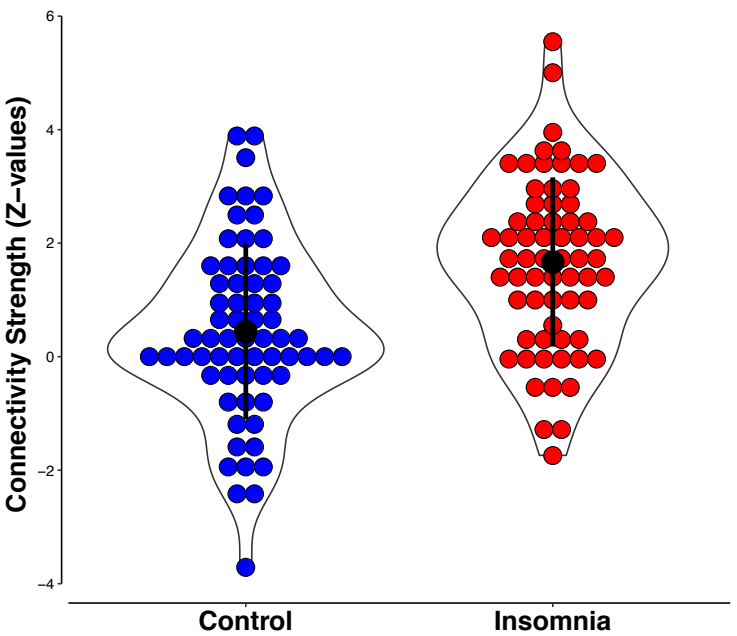

Figure 1. Increased functional connectivity between the bilateral hippocampus and the left middle frontal gyrus in Insomnia Disorder. A) Left middle frontal gyrus cluster (red) showing significantly higher functional connectivity with the bilateral hippocampus in insomnia participants than in controls without sleep complaints ( $p=.035$, cluster based correction for multiple comparisons at $\alpha=.05, Z>$ 3.1). B). The violin plot displays the mean, standard deviation and individual values of the connectivity strength between the hippocampus and the middle frontal gyrus for the participants with insomnia (red) and the controls (blue).
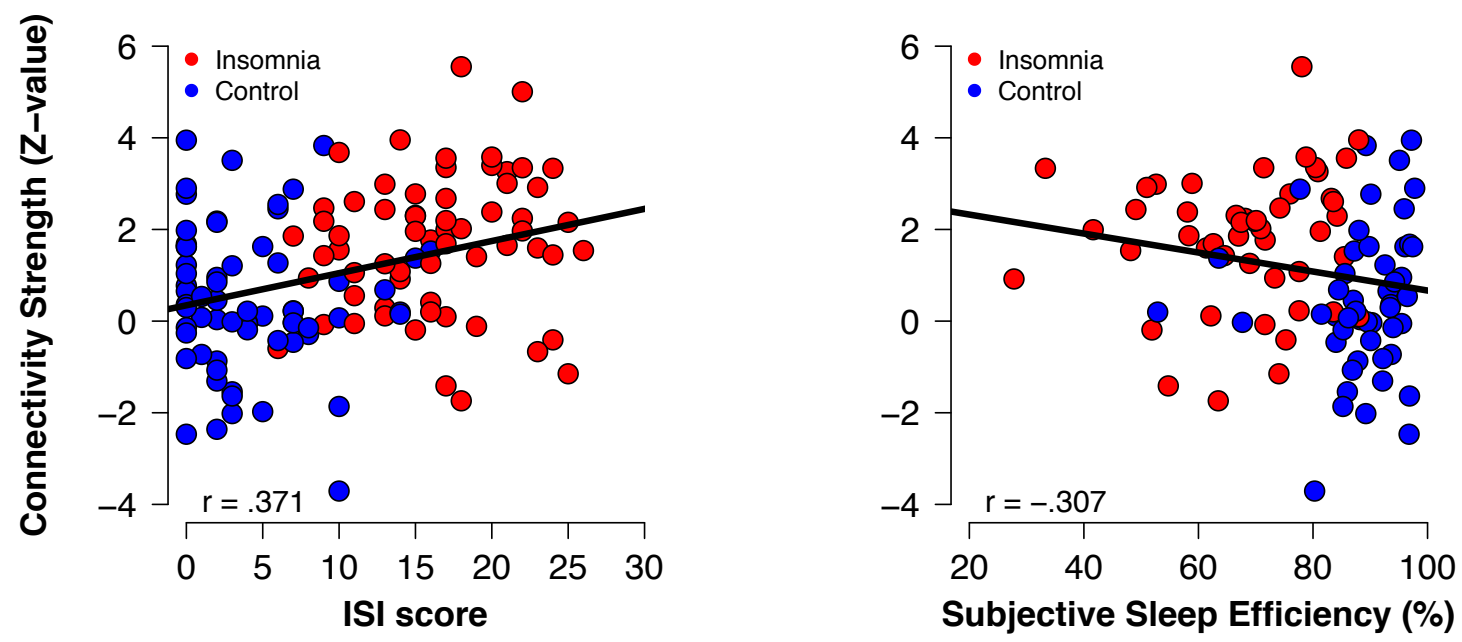

Figure 2. Scatterplots showing how bilateral hippocampus - left middle frontal gyrus (MFG) connectivity strength is associated with subjective Insomnia severity (ISI) and subjective sleep efficiency (Consensus Sleep Diary), for the participants with insomnia (red) and controls (blue). 


\section{Tables}

Table 1. Descriptive Statistics (\% or $M \pm S D$ )

\begin{tabular}{lccc}
\hline & $\begin{array}{c}\text { Insomnia } \\
(\mathrm{N}=65)\end{array}$ & $\begin{array}{c}\text { Control } \\
(\mathrm{N}=65)\end{array}$ & p-value \\
& 26.2 & 35.4 & .342 \\
Sex (\% male) & $48.3 \pm 14.0$ & $44.1 \pm 15.2$ & .108 \\
Age (years) & $20.2 \pm 15.9$ & - & \\
Duration of insomnia (years) & & & \\
Subjective insomnia severity & & & \\
$\quad$ ISI $^{1}$ & $16.3 \pm 5.2$ & $4.2 \pm 4.3$ & $2.2 \mathrm{e}-16^{*}$
\end{tabular}

Subjective sleep (CSD) ${ }^{2}$

$\begin{array}{lccc}\text { Sleep onset latency }(\min ) & 41.5 \pm 40.5 & 15.5 \pm 10.5 & 3.0 \mathrm{e}-05^{*} \\ \text { WASO (min) } & 65.3 \pm 38.1 & 22.2 \pm 24.5 & 1.5 \mathrm{e}-9^{*} \\ \text { Total sleep time (min) } & 322 \pm 71.9 & 426 \pm 52.0 & 8.6 \mathrm{e}-13^{*} \\ \text { Sleep efficiency (\%) } & 68.2 \pm 14.0 & 88.6 \pm 8.6 & 8.1 \mathrm{e}-14^{*}\end{array}$

Objective sleep (PSG) ${ }^{3}$

$\begin{array}{lccc}\text { Sleep onset latency }(\mathrm{min}) & 14.0 \pm 13.5 & 13.9 \pm 18.1 & .972 \\ \text { WASO }(\min ) & 54.4 \pm 43.3 & 33.5 \pm 24.7 & .003^{*} \\ \text { Total sleep time (min) } & 400 \pm 62.0 & 432 \pm 55.5 & .007^{*} \\ \text { Sleep efficiency (\%) } & 83.5 \pm 11.1 & 89.5 \pm 7.1 & .001^{*}\end{array}$

Note: ${ }^{1} N=128\left(N_{I D}=64, \mathrm{~N}_{\text {control }}=64\right),{ }^{2} N=98\left(N_{I D}=48, N_{\text {control }}=50\right),{ }^{3} N=106\left(N_{I D}=\right.$ 51, $\left.N_{\text {control }}=55\right)$. ID = Insomnia Disorder, $M=$ mean, $S D=$ standard deviation, ISI = insomnia severity index, CSD = Consensus Sleep Diary, $\mathrm{PSG}=$ polysomnography, $\mathrm{WASO}=$ wake after sleep onset. * significant at $\alpha<.05$. 
Table 2. Partial Pearson correlation analyses between connectivity strength and subjective and objective sleep parameters

r $\quad$ Uncorrected $\mathrm{p} \quad$ FDR corrected $\mathrm{p}$

Subjective insomnia severity
ISI $^{1}$
.371
$1.0 \mathrm{e}-5^{*}$
$9.3 \mathrm{e}-5^{*}$

Subjective sleep (CSD) ${ }^{2}$

$\begin{array}{lccc}\text { Sleep onset latency }(\mathrm{min}) & .131 & .208 & .268 \\ \text { WASO (min) } & .178 & .083 & .125 \\ \text { Total sleep time (min) } & -.213 & .036^{*} & .082 \\ \text { Sleep efficiency (\%) } & -.307 & .002^{*} & .009^{*}\end{array}$

Objective sleep (PSG) ${ }^{3}$

$\begin{array}{lccc}\text { Sleep onset latency }(\mathrm{min}) & -.027 & .790 & .790 \\ \text { WASO (min) } & .226 & .021^{*} & .062 \\ \text { Total sleep time (min) } & -.056 & .575 & .647 \\ \text { Sleep efficiency (\%) } & -.178 & .070 & .125\end{array}$

Note: Correlations adjusted for age, sex, scanner site, time of day, $r=$ correlation coefficient. ${ }^{1} N=128\left(N_{I D}=64, N_{\text {control }}=64\right),{ }^{2} N=98,\left(N_{I D}=48, N_{\text {control }}=50\right),{ }^{3} N=106$ $\left(N_{I D}=51, N_{\text {control }}=55\right) . \mathrm{ID}=$ Insomnia Disorder, ISI = insomnia severity index, $\mathrm{CSD}=$ Consensus Sleep Diary, PSG = polysomnography, WASO= wake after sleep onset. * significant at $\alpha<.05$. 